\title{
Nickel Allergy In Orthodontics
}

Vijay Naik ${ }^{1}$, Kinjal Mavani²

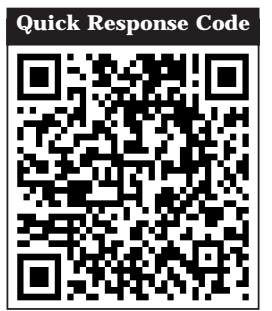

doi: $10.5866 / 2015.7 .10259$

1Professor \& Head

${ }^{2}$ Post graduate student

Department of Orthodontics and Dentofacial

Orthopedics,

Maratha Mandal's Nathajirao G. Halgekar Institute of

Dental Sciences and Research Centre, Belgaum

\section{Article Info:}

Received: October 7, 2015

Review Completed: November 8, 2015

Accepted: December 10, 2015

Available Online: March, 2016 (www.nacd.in)

(C) NAD, 2015 - All rights reserved

Email for correspondence:

drvijay_naik@rediffmail.com

\begin{abstract}
:
Nickel is a common component in many orthodontic materials. An allergy to nickel is commonly seen in the population, more frequently in women. This allergy has increased with the more frequent use of nickel containing jewellery and intraoral piercings. As a result, this allergy can be expected to be more readily encountered in dental practice. Possibleallergy to nickel should be a question in the initial patient health history questionnaire. The dental practitioner should be mindful of this allergy during the course of orthodontic treatment, and know how to diagnose a nickel allergy if it appears and subsequent action in treatment and referral if it is suspected. This paper provides a summary of nickel allergy, its epidemiology, diagnosis and recommendations and alternatives to treatment.
\end{abstract}

Key words: Allergy; Nickel hypersensitivity; Orthodontics;

\section{Introduction}

Nickel allergy is by far the most common contact allergy in the industrial world, with a prevalence of up to $30 \%$, depending on age, sex, and ethnicity. ${ }^{1,2}$ Possible sources for nickel sensitization include jewellery, clothing fasteners, mobile phones, and, in particular, (ear) piercings.

Nickel allergy a concern for the orthodontist because it is present in a vast array of materials frequently used in orthodontics. Nickel is the most common component of the super-elastic nickeltitanium ( $\mathrm{Ni}-\mathrm{Ti})$ archwires used during the initial levelling and aligning phase of orthodontic treatment with a concentration of $47-50 \%{ }^{3}$ It is also a component in stainless steel (present in both archwires and brackets), representing approximately $8 \%$ of the alloy. Extraoral orthodontic appliances such as the outer bows of headgears contain nickel and may also elicit a response on the skin. ${ }^{4}$

\section{Biology and Immune Response of Nickel Allergy}

The response by the immune system to nickel is usually a Type IV cell mediated delayed hypersensitivity also called an allergic contact dermatitis. It is mediated by T-cells and monocytes/ macrophages rather than antibodies and consists of two phases. The first phase, or sensitisation, occurs when nickel initially enters the body. There is usually no response present at this time but the immune system is primed or sensitised for an allergic response. The major sensitisation routes are 
nickel-containing jewellery and foods. Foods that are high in nickel includechocolate, soy beans, nuts and oatmeal. A response, or the elicitation phase, is in the form of a contact mucositis or dermatitis that occurs during re-exposure to nickel and develops over a period of days or rarely up to three weeks. If nickel is leached from orthodontic appliances, this Type IV hypersensitivity reaction cans occur. ., $^{6}$

\section{Prevalence of Nickel Allergy}

Nickel allergy occurs more frequently than allergy to all other metals combined. ${ }^{5}$ The incidence of $\mathrm{Ni}$ induced side effects from orthodontic materials in non-sensitized people is not known. It has also been suggested that the risk of sensitization from orthodontically derived $\mathrm{Ni}$ in these patients is extremely low. Nickel allergy is the most common contact allergy in industrialized countries; patch test verified data of general populations in several studies have shown that this allergy affects 10\%30\% of females and $13 \%$ of males. ${ }^{7,8}$

This is thought to be due to ear piercing being a major cause of sensitization to nickel, as prevalence in subjects with pierced ears was $31 \%$ and those without pierced ears was $2 \% .{ }^{9}$ People with cutaneous piercing were considered a significant risk factor for $\mathrm{Ni}$ allergy. Scientific evidence suggests that orthodontic treatment is not associated with increase of $\mathrm{Ni}$

Hypersensitivity, unless patients have a history of previous cutaneous piercing exposure to $\mathrm{Ni}$, usually ear piercing. Previous allergic history has been significantly associated by several authors to a hypersensitivity response to nickel released from orthodontic appliances.

Fortunately, most individuals who have nickel sensitivity do not report adverse clinical manifestations to orthodontic appliances containing nickel. It is estimated that the occurrence of a harmful response by patients to nickel is $0.1-0.2 \%{ }^{10}$ It is thought that a much greater concentration of nickel in the oral mucosa than the skin is necessary to elicit an allergic reaction. ${ }^{11} \mathrm{Nickel}$ leaching of orthodontic bands, brackets and stainless steel or $\mathrm{Ni}-\mathrm{Ti}$ arch-wires has been shown in vitro to maximally occur within the first week and then decline thereafter. This coincides with the approximatetimeframefor Type IV hypersensitivity reactions. Saliva or certain intraoral conditions such as foods, oral hygiene products and fluoride may potentially corrodethe nickel in thealloy and rel ease it onto the oral mucosa.

\section{Clinical Features Associated With Allergy}

Oral clinical signs and symptoms of nickel allergy can include the following: a burning sensation, gingival hyperplasia, labial desquamation, angular chelitis, erythema multiforme, periodontitis, stomatitis with mild to severeerythema, papularperi-oral rash, loss of taste or metallic taste, numbness, soreness at side of the tongue. ${ }^{12}$

These reactions are associated with an inflammatory response induced by corrosion of orthodontic appliances and subsequent release of nickel. It is manifested as Nickel Allergic Contact Stomatitis (NiACS). A burning sensation is the most frequent symptom. The aspect of the affected mucosa is also variable, from slight erythema to shiny lesions, with or without edema. In chronic cases, the affected mucosa is typically in contact with the causal agent and appears erythematous or hyperkeratotictoulcerated.Extraoral manifestations of nickel allergy may have an intraoral origin. ${ }^{13}$

\section{DIAGNOSIS}

Sensitivity to nickel has been evaluated through biocompatibility tests, including cutaneous sensitivity (patch) tests, and reactivity to nickel has been evaluated with in vitro cell proliferation assays.The diagnosis of a response to nickel in the oral mucosa is more difficult than on the skin.It is important to make correct diagnosis of nickel allergy, symptoms of which may occur either within or remote to the oral environment. The signs and symptoms of nickel allergy are presented in Table 1.

The following patient history would suggest a diagnosis of nickel allergy. ${ }^{14}$

1. Previous allergic response after wearing earrings or a metal watchstrap;

2. A known allergy to nickel should be determined when the patient completes the medical questionnaire or during a verbal medical history review 
3. Appearance of allergy symptoms shortly after initial insertion of orthodontic components containing nickel;

4. Confined extra-oral rash adjacent to headgear studs.

A dermatologist should confirm the diagnosis by patch test in using $5 \%$ nickel sulfate in petroleum jelly.

A known allergy to nickel should be determined when the patient completes the medical questionnaire or during a verbal medical history review. The patient should then be forewarned of a possible response to the nickel in orthodontic appliances, particularly to the initial arch-wire placed.

\section{MANAGE MENT OF NICKEL ALLERGY}

\section{Alternatives to Nickel-Titanium Wires}

If intra-oral signs and symptoms are present and a diagnosis of nickel hypersensitivity is established, the nickel titanium arch-wire should be removed and replaced. Alternatives include:

1. Twist flex stainless steel

2. Fiber reinforced composite arch-wires

3. Wires such as TMA, pure titanium, and goldplated wires may also be used without risk.

4. Altered nickel-titanium arch-wires also exist and include plastic/resin coated nickel-titanium archwires. ${ }^{15}$

5. I on-implanted nickel-titanium archwires have their surface bombarded with nitrogen ions, which forms an amorphous surface layer, conferring corrosion resistance and displacing nickel atoms, and decreasing the risk of an allergic response.

Stainless steel is slightly less expensive than $\mathrm{Ni}-\mathrm{Ti}$ archwires while TMA is slightly more. Resin coated $\mathrm{Ni}-\mathrm{Ti}$ wires are also an option. These resincoated wires have had their surface treated with nitrogen ions, which forms an amorphous surface layer. Manufacturers claim that this results in an increase in corrosion resistance and decreased amount of leaching of nickel, more so than both $\mathrm{Ni}$ $\mathrm{Ti}$ and stainless steel wires. ${ }^{15}$

Most patients who develop a reaction to $\mathrm{Ni}-\mathrm{Ti}$ archwires subsequently tolerate stainless steel without a reaction. This is believed to be a result of the nickel being tightly bound to the crystal lattice of the alloy, rendering them unable to be leached into the oral cavity. Stainless steel has been shown to release low amounts of nickel in artificial saliva or sweat which could help account for its low allergenicity. ${ }^{17}$

Incase, the patient continues to manifest an allergic reaction, the patient should be referred to a physician to be treated with antihistaminics, anaesthetics or topical corticosteroids. ${ }^{18}$

Attempts should be made to complete orthodontic treatment with TMA, fibre-reinforced composite, pure Ti or gold-plated wires.

\section{Alternatives to Brackets}

Stainless steel brackets are generally considered safe. However, nickel free alternative brackets to stainless steel include:

1. Ceramic brackets produced using polycrystallinealumina, single crystal sapphire, and zirconia;

Table 1: Signs and symptoms of nickel allergy ${ }^{14}$

\begin{tabular}{ll} 
Intraoral & Extraoral \\
Stomatitis from mild to severe erythema & Generalized urticaria \\
\hline Papula-perioral rash & Widespread eczema \\
\hline Loss of taste or metallic taste & Flareup of allergic dermatitis \\
\hline Burning sensation & Exacerbation of preexisting eczema \\
\hline Soreness at the side of the tongue & \\
\hline Angular cheilitis & \\
\hline Severe gingivitis in the absence of plaque & \\
\hline
\end{tabular}


2. Polycarbonate brackets that are produced from plastic polymers;

3. Titanium brackets;

4. Goldplated brackets;

5. Plastic aligners such as I nvisalign ${ }^{\mathrm{TM}}$.

Extraoral metal components, including metal studs in headgear, are of greatest concern due to greater sensitivity of skin. Plastic coated headgear studs may be a better alternative for such patients.

The majority of investigations have found that nickel sensitive patients areabletotoleratestainless steel without any noticeable reaction. ${ }^{16}$ Most research concludes that stainless steel is a safe material to use for all intraoral orthodontic components for nickel sensitive patients.

\section{CONCLUSION}

Though an allergic response in the oral mucosa due to the nickel content from nickel containing orthodontic appliances is more infrequent than from nickel contact on the epidermis, it can still occur. If nickel-related intraoral clinical signs and symptoms appear, the orthodontist should be prepared to undertake or continue treatment without the use of $\mathrm{Ni}-\mathrm{Ti}$ wires and even without stainless steel. . It is imperative for a practitioner to not only know the physical and mechanical properties of the materials being used, but also of the biologic compatibility of the material. Awareness of reactions that can occur with the various dental materials in treatment is important to the orthodontist. These materials can produce type IV hypersensitivity reactions. Diagnosis and treatment should include a multidisciplinary team. In all instances, the patient's well-being should guide treatment decisions, and general health not just oral health should be the goal. Nevertheless when clinical signs and symptoms presumed to be due to nickel hypersensitivity are distressing to the patients, there are many alternatives available for the orthodontist. Safe and effective practice depends on identifying patients with allergy along with knowledge of materials that can potentially cause them.

\section{REFERENCES:}

1. Rietschel R L, Fowler J F, Warshaw E M et al. Detection of nickel sensitivity has increased in North American patchtest patients. Dermatitis 2008; 19:16-19.
2. Simonsen A B, Deleuran $M$, J ohansen J D, Sommerlund $M$. Contact allergy and allergic contact dermatitis in children - a review of current data. Contact Dermatitis 2011; 65:254265.

3. Eliades T, Athanasiou A E. In vivo aging of orthodontic alloys: implications for corrosion potential, nickel release, and biocompatibility. Angle Orthod 2002; 72: 222-227.

4. Burden D J , Eedy D J . Orthodontic headgear related to allergic contact dermatitis: a case report. Br Dent J 1991; 170: $447-448$.

5. Lowey M N. Allergic contact dermatitis associated with the use of Interlandi headgear in a patient with a history of atopy. Br Dent J 1993; 17: 67-72.

6. Hostynek J J . Sensitization to nickel: etiology, epidemiology, immune reactions, prevention, and therapy. Rev Environ Health 2006; 21:253-280.

7. J anson GR, Dainesi EA, Pereira AC, Pinzan A. Clinical evaluationof nickel hypersensitivity reaction in patients under orthodontic treatment. Ortodontia 1994; 27:317.

8. J acobsen N, HenstenPettersen A. Changes in occupational health problems and adverse patient reactions in orthodontics from 1987 to 2000. E ur J Orthod 2003; 25:5918.

9. Kerosuo H, Kullaa A, Kerosuo E, Kanerva L, HenstenPettersen A. Nickelallergy in adolescents in relation to orthodontic treatment and piercing of ears. Am J Orthod Dentofac Orthop 1996; 109:14854.

10. Menne T. Quantitative aspects of nickel dermatitis: sensitization and eliciting threshold concentrations. Sci Total Environ 1994; 148:275-281.

11. Dunlap C L, Vincent S K, Barker B F. Allergic reaction to orthodontic wire: report of a case. J Am Dent Assoc1989; 118:449-450.

12. Starkjaer L, Menne T. Nickel allergy and orthodontic treatment. Eur J Orthod1990; 12:284-289.

13. SchultzJ C, Connelly E, Glesne L, Warshaw E M. Cutaneous and oral eruption from oral exposure to nickel in dental braces. Dermatitis 2004; 15:154-157.

14. Rahilly G, PriceN. Nickel allergy and orthodontics.J Orthod 2003; 30:1714.

15. Kim H, J ohnson J. Corrosion of stainless steel, nickel titanium, coated nickel titanium, and titanium orthodontic wire. Angle Orthod 1999; 69:3944.

16. Toms A P. The corrosion of orthodontic wire. Eur J Orthod1988; 10: 87-97.

17. J ensen C S, Lisby S, Baadsgaard O, Byrialsen K, Menné T. Release of nickel ions from stainless steel alloys used in dental braces and their patch test reactivity in nickelsensitive individuals. Contact Dermatitis 2003; 48:300-304.

18. Dou X, Liu L L, Zhu X J . Nickel-elicited systemic contact dermatitis. Contact Dermatitis 2003; 48:126-129. 